Año 16, Vol. 11, número 21, agosto-diciembre 2021

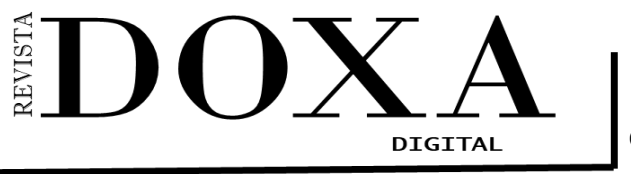

eISSN: 2594-2786

Sección: Dossier

Ponencia presentada el 1 de junio del 2021

DOI: $10.52191 /$ rdojs.2021.243

Pág.: 143-152

Ponencia presentada en la mesa 1:

"Estudios de pregrado en comunicación, organizaciones y gestión"

Primer Coloquio de Estudiantes y Egresados de Licenciatura y Posgrado

Ciudad Juárez, México 1 y 2 de junio 2021

\title{
COVID-19 y logística: cambios de tendencia, retos disciplinares y prácticos
} COVID-19 and logistics: Changing trends, disciplinary and practical challenges

María Del Carmen De La Hoz Orozco *, Carlos Alberto Rodríguez Arias ** y Julian Garib Barrera Gonzalez ***

\section{RESUMEN}

La pandemia causada por el Covid-19 ha generado cambios importantes en las sociedades; Desde estas nuevas formas para la logística, los cambios no solo se evidencian en las nuevas formas que se desarrollan los procesos logísticos, sino también desde su campo de estudio, teniendo en cuenta que los avances tecnológicos, específicamente en logística 4.0, son el presente que antes de pandemia, se consideraba futuro. Así las cosas, el objetivo de este artículo es analizar los cambios de tendencia, retos disciplinares y prácticos causados por la pandemia en el área de logística. A través de un análisis cienciometrico y hermenéutico, que tenga en cuenta un periodo antes de pandemia 2010-2019 y un periodo durante pandemia 2020, se analizarán los distintos cambios desde el campo disciplinar y práctico del área de estudio. Los resultados principales develan que los cambios de la sociedad, acelerados por la pandemia, hacia una economía digital ha obligado a las empresas a hacer ajustes en sus procesos teniendo como base las tecnologías 4.0 que permitan su adaptación a los cambios en los mercados. Finalmente, desde los retos a concebir de la logística se encuentra la preparación de las empresas en la adopción de nuevas tecnologías disruptivas que incorporen en sus procesos logístico, no obstante, es importante la sinergia desde la academia para la formación de profesionales que puedan asimilar estas tecnologías e implementarlas a manera de solución en sus empresas.

PALABRAS CLAVE: logística, análisis cienciometrico, mapa bibliométrico, retos disciplinarios

\footnotetext{
* Estudiante del programa de comercio exterior, integrante del semillero de investigación SIET, Corporación Educativa del Litoral - Contacto: delahozmaria@litoral.edu.co

** Autor para correspondencia. Magister en Ciencias de la Administración y las Organizaciones, Docente investigador, grupo de investigación GICEL, Corporación Educativa del Litoral; Investigador, Escuela de Formación de Infanteria de Marina, Armada Nacional - Contacto: crodriguez@litoral.edu.co

*** Magister en Educación, Docente investigador, Grupo de Investigación Baron de Jominí, Escuela de Formación de Infantería de Marina. Contacto: julian.barrera@armada.mil.co
} 
Año 16, Vol. 11, número 21, agosto-diciembre Sección: Dossier

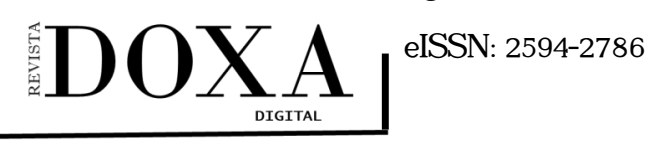

DOI: $10.52191 /$ rdojs.2021.243

Pág.: 143-152
María del Carmen de la Hoz Orozco et al...

COVID-19 y logística...

\section{Introducción}

La pandemia causada por el Covid-19 ha generado cambios importantes en las sociedades, desde aspectos económicos, políticos, sociales y culturales lo que ha traído nuevas formas de ver y actuar sobre las realidades. Desde estas nuevas formas, la gestión logística como proceso que implica garantizar el relacionamiento comercial entre empresas, consumidores y países, también han sufrido afectaciones. Desde esta óptica, se evidencia los trastornos que ha padecido la cadena de suministro y su impacto económico en las empresas y los mercados financieros. La principal causa de esta problemática se debe a las limitaciones en la interacción social, las relaciones internacionales y las cuarentenas obligatorias, que ocasionaron la ralentización de la red de distribución internacional respecto al represamiento de mercancías del exterior, se estima que, en tiempos de pandemia, el comercio internacional tuvo una disminución en la recepción de mercancías de un $40 \%$. A su vez, en la distribución nacional por las restricciones en toda la cadena de valor de los productos y de movilidad, se vio afectado el abastecimiento de productos esenciales.

En este sentido, los cambios no solo se evidencian en las nuevas formas que se desarrollan los procesos logísticos, sino también desde su campo de estudio, teniendo en cuenta que los avances tecnológicos, específicamente en logística 4.0, son el presente que antes de pandemia, se consideraba futuro.

Así las cosas, las consecuencias de la pandemia implica un reto desde la logística como área disciplinar, y es que con los nuevos avances tecnológicos su adaptación a estas nuevas realidades es necesaria.

Dado lo anterior, este artículo de investigación pretende determinar aquellos cambios en la tendencia del área de logística, entendiendo los retos en un contexto de pandemia mundial. Para tal caso, La identificación de tendencias en un campo de investigación sobre logística, se realizaron basadas en análisis bibliométricos y hermenéuticos.

\section{Referente teórico}

En el mundo empresarial y comercial, la logística ha ido evolucionando con la historia y ha mantenido la misma posición para funciones muy diferentes. Esto ha creado confusión, al haber empleado muchos términos: operaciones, supply chain, cadena de suministro, aprovisionamiento, transporte, etc. El nacimiento de la Logista se remonta entre los años 2.900 al 2.800 a.c. donde el ser humano almacenaba comida en las cuevas con el propósito de tener comida durante el invierno 
Año 16, Vol. 11, número 21, agosto-diciembre Sección: Dossier

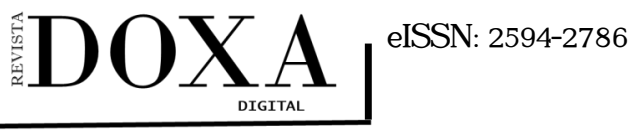

DOI: $10.52191 /$ rdojs.2021.243

Pág.:143-152
María del Carmen de la Hoz Orozco et al...

COVID-19 y logistica..

y aquellas estaciones que se les dificultara salir en busca de su alimento, gestionando lo que hoy día conocemos como el proceso de aprovisionamiento y el control de inventarios. Así mismo, se concibe desde los tiempos de guerra en el cual se enfrentaban distintas facciones y era necesario organizar la logística para el aprovisionamiento de suministros y materiales para los ejércitos (Mora, 2010)

En los años 80 comienza a cobrar verdadera relevancia los tiempos de entrega y la gestión de materiales, así como el servicio al cliente. Para ello, se aumentó la productividad de las plantas distribuidoras y se racionalizaron correctamente los servicios de transporte, teniendo en cuenta que la aparición de la globalización, el aumento de las operaciones a nivel internacional con llevaba a la integración y el control de todas las operaciones (Mora, 2010).

Según Ocampo (2009), el objetivo de la logística es aumentar las ventajas competitivas, captando y reteniendo clientes y generando un incremento en los beneficios económicos obtenidos por la comercialización y producción de los bienes y servicios, mediante la interacción de las actividades mencionadas anteriormente: distribución física, aprovisionamiento de materias primas, manejo de información, tiempos de respuesta, control de nivel de inventarios, estudio de la demanda, servicio al cliente. Todo ello se traduce en una tasa de retorno de la inversión más elevada, con un aumento de la rentabilidad.

Para complementar la logística junto a la cadena de suministros busca gerenciar estratégicamente la adquisición, el movimiento, el almacenamiento de productos y el control de los inventarios, así como todo el flujo de información asociado, a través de los cuales la organización y su canal de distribución se encauzan de tal modo que la rentabilidad presente y futura de la empresa es maximizada en términos de costes y efectividad. De esta forma hoy podemos entender a la logística como la organización de las actividades de aprovisionamiento de materias primas, productos semielaborados y componentes, desde las fuentes de suministro a los centros de producción y de estos, ya transformadas por el proceso productivo, a las plataformas o almacenes de distribución de forma que lleguen al mercado en perfectas condiciones y con un coste mínimo.

\section{Diseño metodológico}

La identificación de tendencias en un campo de investigación sobre gestión logística, se realizó basado en análisis bibliométricos. Para la realización de este análisis se empleó la técnica de análisis 
Año 16, Vol. 11, número 21, agosto-diciembre Sección: Dossier

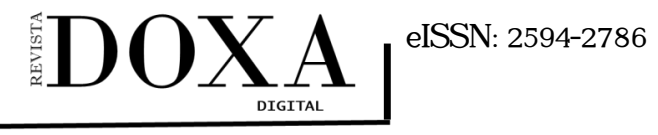

DOI: $10.52191 /$ rdojs.2021.243

Pág.:143-152
María del Carmen de la Hoz Orozco et al...

COVID-19 y logística...

temporal, con el fin de mapear el campo de investigación de la gestión logística para el año 20102019 y 2020. En el análisis realizado, es importante destacar la presencia de nodos y clúster en el mapa bibliométrico, para el primer caso, estos serán las diferentes temáticas que se presentan en el periodo analizado. Por otra parte, el clúster representan la sumatoria de distintos nodos que tienen relaciones entre sí, es decir, esas temáticas se han estudiado de manera agrupada y no separada, por lo tanto demuestran tendencias investigativas desde el análisis del mapa bibliométrico (Garfield, 1994; McKerlich, Ives, \& McGreal, 2013; Price \& Gürsey, 1975; Small, 1993). Este análisis temporal se combinará con un análisis hermenéutico de las relaciones entre los resultados arrojados por la interrelación de clústeres y nodos representativos.

Para la definición de asociaciones entre nodos se utilizó el método de fuerza de asociación (Callon, Courtial, Turner, \& Bauin, 1983; Van Eck \& Waltman, 2007), así mismo, para la identificación de nodos más relevantes. Cabe resaltar que se utilizó como fuente de datos bibliométricos la base SCOPUS por su importancia y nivel de información detallada desde este ámbito.

\section{$\underline{\text { Datos }}$}

Teniendo en cuenta el alcance del primer objetivo es importante determinar los cambios de tendencia en investigación desde la gestión logística entre los años de 2010-2019 y 2020, a través de la base de datos SCOPUS, se delimitó la búsqueda con las palabras claves, "International Business" así mismo por área de conocimiento la cual fue: "Business, Management and Accouting" y "Economics, Econometrics and Finance". Esto permitió delimitar mucho más la búsqueda para ser más eficiente en la selección de artículos relacionados al campo de estudio de los negocios internacionales. En total resultaron 1547 documentos para el periodo 2010-2019 y 152 documentos publicados en el 2020.

Una vez realizado esto, el preprocesamiento de los datos se realizó una vez extraído la información de la base de datos de SCOPUS, se tuvo en cuenta la detección de duplicados y errores en la escritura de palabras claves, autores y revistas (Garfield, 1994; McKerlich et al., 2013).

\section{Resultados}

\section{$\underline{\text { Tendencias desde el campo de investigación en gestión logística }}$}

Las tendencias desde el campo de investigación en gestión logística para el año 2010-2019 se evidencian en la figura 1. En esta se identifican 6 clúster y 73 nodos, es decir, 6 tendencias en investi- 
Año 16, Vol. 11, número 21, agosto-diciembre Sección: Dossier

DOXA
DOI: $10.52191 /$ rdojs.2021.243

Pág.:143-152
María del Carmen de la Hoz Orozco et al... COVID-19 y logística...

gación y 73 temas interrelacionados entre sí.

Figura 1. Análisis temporal: mapa de tendencias en investigación de documentos publicados en los años 2010-2019 en el área de gestión logística

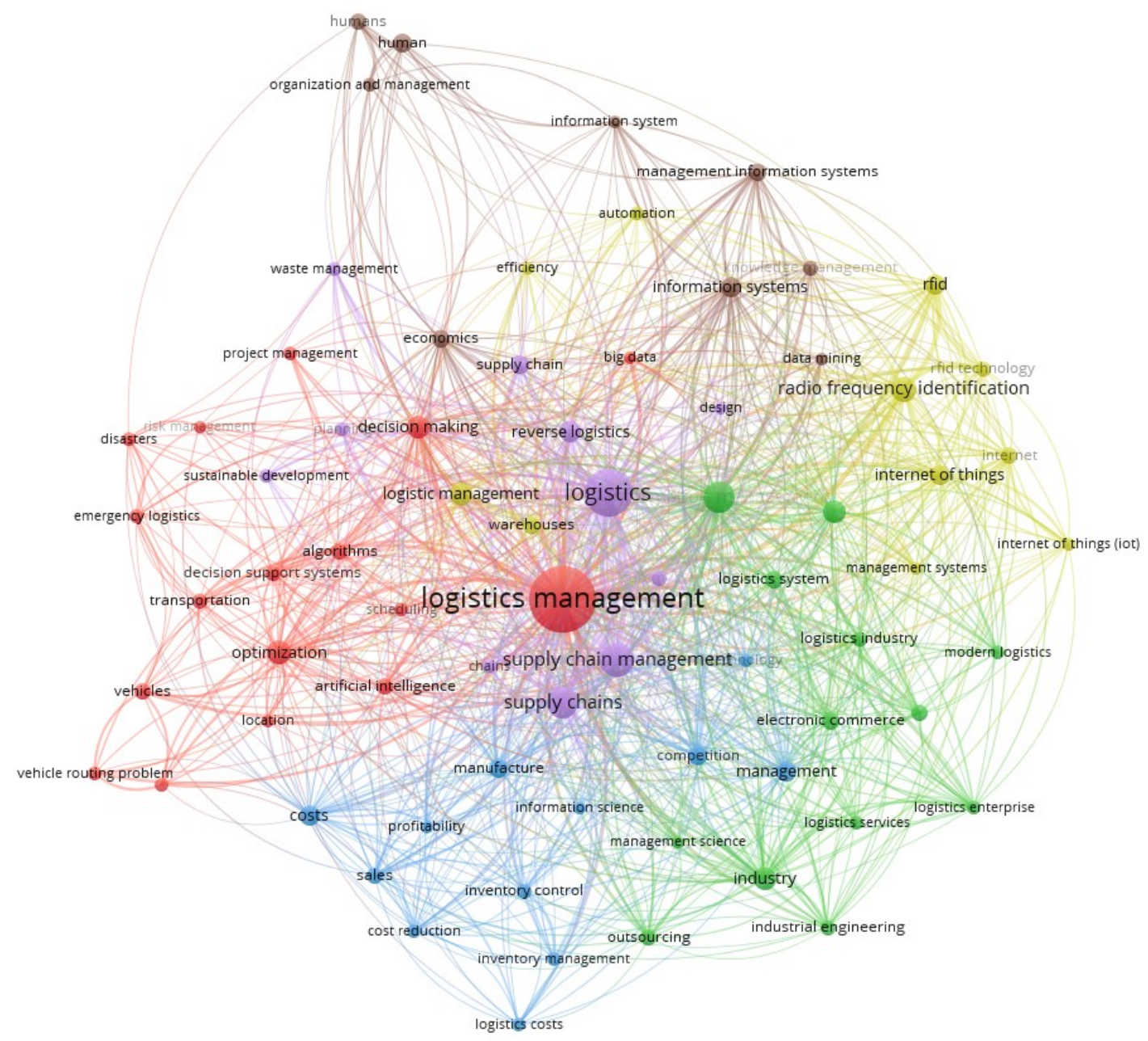

Fuente: Elaboración propia

Para este año, se resaltan estudios diversos desde la gestión logística, es decir no se destacan temáticas individuales sino agrupadas, por lo cual es importante analizar desde las tendencias o clúster formados. Así las cosas, desde el clúster rojo se identifican tendencias en investigación relacionadas con la optimización en todo el proceso logístico, principalmente desde el transporte, enfatizando el uso de inteligencia artificial. No es de extrañar en este caso, temáticas secundarias como algoritmos, problemas de ruteo, big data y analítica de datos. Es importante destacar desde el 
Año 16, Vol. 11, número 21, agosto-diciembre Sección: Dossier

\section{DOXA}

eISSN: 2594-2786

DOI: $10.52191 /$ rdojs.2021.243

Pág.: 143-152
María del Carmen de la Hoz Orozco et al...

COVID-19 y logística..

clúster verde las tendencias en investigación sobre las distintas variantes de la logística estudiada en conjunto (logísticas de servicios, logística empresarial, logística industrial, sistemas logísticos entre otros). Por otro lado, se evidencia desde el clúster amarillo, aquellas tendencias relacionadas con internet de las cosas e identificación de radio frecuencia, esto relacionado con las temáticas secundarias sobre eficiencia y automatización, los cuales se encuentran estrechamente ligadas a las primarias.

Finalmente, el clúster morado que de acuerdo a su posición es el de mayor centralidad en el mapa bibliométrico, lo que indica mayor interrelación con los demás clúster y temáticas asociadas. Estos los representan estudios asociados a la cadena de suministro principalmente, mientras que temáticas secundarias asociadas a el desarrollo sostenible.

Para el caso del año 2020 las tendencias varían con respecto al año 2019, es importante destacar que en este periodo presentan 3 clúster con 15 temáticas asociadas a esto.

Figura 2. Análisis temporal: mapa de tendencias en investigación de documentos publicados en el año 2020 en el área de gestión logística

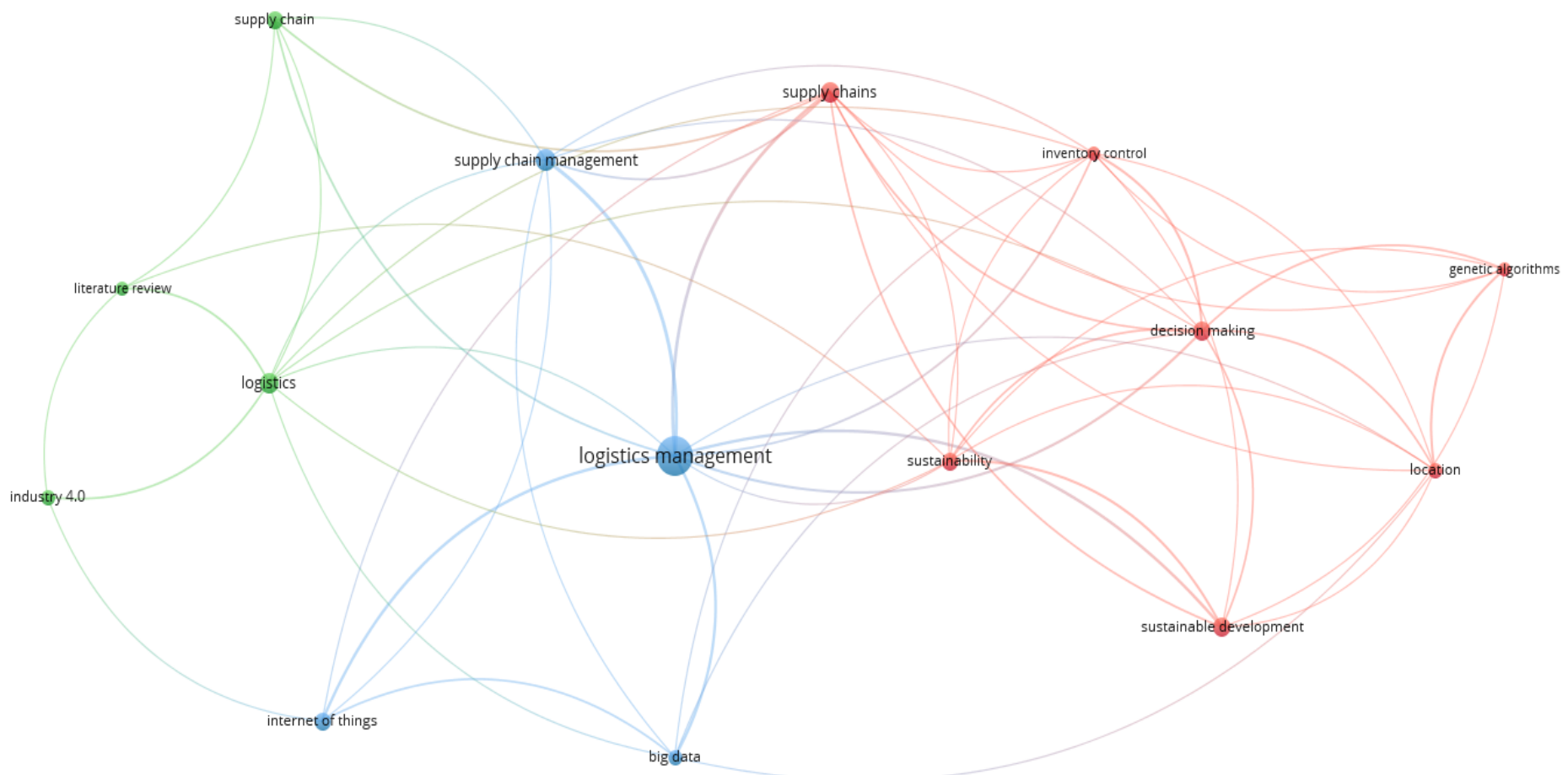

Fuente: Elaboración propia 
Año 16, Vol. 11, número 21, agosto-diciembre Sección: Dossier

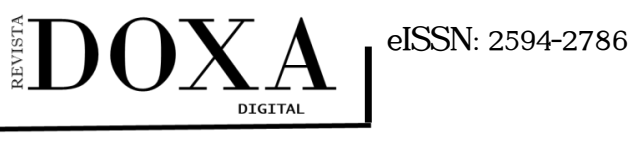

DOI: $10.52191 /$ rdojs.2021.243

Pág.: 143-152
María del Carmen de la Hoz Orozco et al...

COVID-19 y logística...

Respecto a la figura 2, es importante destacar el clúster azul el cual está asociado al internet de las cosas y big data combinado con la gestión de cadena de suministro, es importante destacar que estas temáticas tienen presencia desde el año 2010-2019. Por otra parte, el clúster rojo, tiene presencias temáticas desde la sostenibilidad y el desarrollo sostenible. Finalmente, el clúster verde representa la industria 4.0 y la cadena de suministro.

Desde estos hallazgos, es importante establecer que la logística como campo de estudio enfrenta retos en el marco de la pandemia, estos retos van desde las prácticas de gestión logística para empresas y países, así mismo para la formación de profesionales en áreas de logística.

En este sentido, para los retos de las empresas y países se concibe:

- Crecimiento del comercio electrónico o E-Supply Chain Managemenet": con el auge del comercio electrónico, es relevante que se rediseñen los procesos de la cadena de suministro sobre todo en almacenaje y transporte (Sánchez, Pérez, Sangroni, Cruz, \& Medina, 2021)

- Evolución de los clientes: por las nuevas tecnologías, las respuestas rápidas y flexibles que deben dar las empresas requiere una mayor eficiencia en las cadenas logísticas de las empresas (Sánchez et al., 2021)

- Digitalización de los procesos: desarrollo de competencias en el talento humano de las empresas que permitan adoptar las nuevas tecnologías (Sánchez et al., 2021)

- Cambios tecnológicos: adopción de tecnologías disruptivas, 5G, internet de las cosas Analítica de datos, machine learning (Sánchez et al., 2021).

Por su parte desde la academia, todos estos cambios implican también la preparación de profesionales idóneos ante los nuevos retos que obedece la coyuntura:

- Multidisciplinar: que contemple la integración de múltiples disciplinas y las tecnologías para la resolución de problemas de empresas y países.

- Habilidades digitales: sin duda la gran conclusión de la pandemia es que todos los actores, sin excepción, debemos poder desarrollar habilidades digitales desde, softwares de gestión hasta tecnologías de realidad virtual o realidad aumentada para generar procesos eficientes.

- $\quad$ Analista de datos: incluyendo la big data, analítica de datos e inteligencia artificial para la 
Pág.: 143-152

toma de decisiones en tiempo real y ajustada a las necesidades del sector.

\section{Conclusiones}

La gestión logística implica definir adecuadamente, acorde al contexto, los procesos de intercambio de bienes y servicios entre empresas y países. Su importancia radica en la optimización de los procesos logístico que mejoren la eficiencia de los actores. Desde los estudios asociados a la gestión logística, existen tendencias transversales como la sustentabilidad y sostenibilidad de los procesos, conocida en ingles como "Green Supply Chain Management", así mismo la preocupación constante para definir los procesos de optimización en las empresas a través de tecnologías 4.0 se desarrolla en cada periodo analizado. El resumen de las tendencias puede observarse en la tabla 1 y 2 :

Tabla 1. Tendencias en investigación para el año 2019 - Gestión Logística

\begin{tabular}{|c|c|c|}
\hline Periodo & Principales Temáticas & Temáticas Secundarias \\
\hline Clúster Rojo & $\begin{array}{l}\text { Optimización; big data; inteligencia arti- } \\
\text { ficial }\end{array}$ & \multirow{4}{*}{$\begin{array}{c}\text { Transporte; Toma de decisiones; gestión de pro- } \\
\text { yectos; problemas de ruteo de vehículos } \\
\text { Gestión de la información; Logística moderna; Co- } \\
\text { mercio electrónico } \\
\text { Administración; Costos; Ventas; Rentabilidad } \\
\text { RFID; Automatización; identificación de radio fre- } \\
\text { cuencias }\end{array}$} \\
\hline Clúster Verde & Sistemas logísticos; industria & \\
\hline Clúster azul & Costos logísticos; Manufactura & \\
\hline Clúster Amarillo & Internet de las cosas; Eficiencia & \\
\hline Clúster Morado & $\begin{array}{c}\text { Gestión de la cadena de suministro; } \\
\text { Sostenibilidad }\end{array}$ & \multirow{2}{*}{$\begin{array}{l}\text { Cadena de suministro; Logística inversa } \\
\text { Economía; minería de datos; administración y or- } \\
\text { ganización }\end{array}$} \\
\hline Clúster Marrón & Sistemas de información & \\
\hline
\end{tabular}

Fuente: elaboración propia

Tabla 2. Tendencias en investigación para el año 2020 - Gestión Logística

\begin{tabular}{ccc}
\hline Periodo & Principales Temáticas & Temáticas Secundarias \\
\hline Clúster azul & Gestión de la cadena de suministro & Internet de las cosas; Analítica de datos \\
Clúster Verde & Industria 4.0 & Cadena de suministro \\
Clúster Rojo & Sostenibilidad; Desarrollo Sostenible & Localidad; algoritmos \\
\hline
\end{tabular}

Fuente: elaboración propia

Es importante destacar que los cambios de la sociedad, acelerados por la pandemia, hacia una economía digital ha obligado a las empresas a hacer ajustes en sus procesos teniendo como base las tecnologías 4.0 que permitan su adaptación a los cambios en los mercados. En este sentido, Taghipour (2021) propone una nueva variante desde la gestión logística: "E-Supply Chain Mana- 
Año 16, Vol. 11, número 21, agosto-diciembre Sección: Dossier

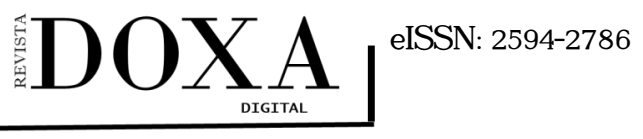

DOI: 10.52191/rdojs.2021.243

Pág.:143-152
María del Carmen de la Hoz Orozco et al...

COVID-19 y logística..

gemenet", el cual propone la sinergia entre los mercados electrónicos y la gestión logística a través de la gestión de la cadena de suministro.

Finalmente, desde los retos a concebir de la logística se encuentra la preparación de las empresas en la adopción de nuevas tecnologías disruptivas que incorporen en sus procesos logístico, no obstante, es importante la sinergia desde la academia para la formación de profesionales que puedan asimilar estas tecnologías e implementarlas a manera de solución en sus empresas.

\section{Referencias bibliográficas}

Callon, M., Courtial, J.-P., Turner, W. A., \& Bauin, S. (1983). From translations to problematic networks: An introduction to co-word analysis. Social Science Information, 22(2), 191-235. https:// doi.org/10.1177/053901883022002003

Garfield, E. (1994). Scientography: Mapping the tracks of science. Current Contents: Social \& Behavioural Sciences, 7(45), 5-10.

McKerlich, R., Ives, C., \& McGreal, R. (2013). Measuring use and creation of open educational resources in higher education. International Review of Research in Open and Distance Learning, 14(4), 90-103. https://doi.org/10.1002/asi

Mora, L. (2010). Gestión logística integral: Las mejores prácticas en la cadena de abastecimiento. In Editorial EcoeEdiciones. Retrieved from https://www.ecoeediciones.com/wp-content/ uploads/2016/12/Gestion-logistica-integral-2da-Edición.pdf

Price, D. de S., \& Gürsey, S. (1975). Studies in scientometrics I: Transience and continuance in scientific authorship. Ciência Da Informação, 4(1), 27-40. https://doi.org/10.1007/s11434-0135939-3

Sánchez, Y., Pérez, J., Sangroni, N., Cruz, C., \& Medina, Y. (2021). Retos actuales de la logística y la cadena de suministro Current challenges of logistics and supply chain. Ingeniería Industrial, XLII(1), 1-12.

Small, H. (1993). Macro-Level Changes in the structure of co-citation clusters: 1983-1989. Sciencometrics, 26(1), 5-20. https://doi.org/10.1097/00006324-197504000-00010

Taghipour, A. (2021). E-Supply Chain Management : A review E-Supply Chain Management : A re- 
Año 16, Vol. 11, número 21, agosto-diciembre Sección: Dossier

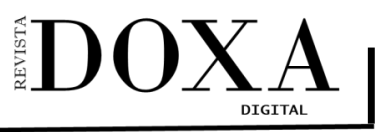

María del Carmen de la Hoz Orozco et al...

COVID-19 y logística...

Pág.:143-152

view. International Journal of E-Education e-Business e-Management and e-Learning, (October).

Van Eck, N. J., \& Waltman, L. (2007). Bibliometric mapping of the computational intelligence field. International Journal of Uncertainty Fuzziness and Knowledge-Based Systems, 15(5), 625-645. https://doi.org/Doi 10.1142/S0218488507004911 